Pacific Journal of Mathematic 


\section{MAPPING PROPERTIES, GROWTH, AND UNIQUENESS OF VIETA (INFINITE COSINE) PRODUCTS}

\section{KeNNETH B. STOLARSKY}

The natural logarithm of $z$ can be written as an infinite product involving iterated square roots of $z$. A Vieta product is defined to be a more general infinite product involving $z$ raised to arbitrary fractional powers. Restricted to the unit circle, Vieta products generalize infinite cosine products studied by Salem and others in connection with $P V$-numbers. Vieta products are shown to have conformal mapping, monotonicity, and growth properties very similar to those of the natural logarithm. By using certain properties of Eulerian polynomials, the exponents of $z$ in a Vieta product are shown to be unique in a strong sense.

1. Introduction. Let $\tau(n)=2^{-n}$. As a simple corollary to his results [2] on logarithmic means, B. C. Carlson obtained

$$
\ln x=(x-1) \prod_{n=1}^{\infty} \frac{2}{1+x^{\tau(n)}}
$$

for $x>0$. This representation immediately implies that $\ln x$ grows more slowly than any power of $x$, and also that

$$
\ln x^{-1}=-\ln x, \quad \ln x^{2}=2 \ln x .
$$

Our aim is to give an extensive, though far from exhaustive, exposition of the properties of infinite products of the form

$$
C=C_{\alpha}(z)=(z-1) \prod_{n=1}^{\infty} \frac{2}{1+z^{\alpha_{n}}}
$$

where $z$ is a complex variable and $\alpha=\left\{\alpha_{n}\right\}$ is a sequence of strictly decreasing positive numbers such that

$$
\sum_{n=1}^{\infty} \alpha_{n}=1 \text {. }
$$

Our main results are that (i) such products have conformal mapping, growth, and monotonicity properties very similar to those of the natural logarithm, especially when the $\alpha_{n}$ decrease geometrically, and that (ii) the function $C_{\alpha}(z)$ uniquely determines the sequence $\left\{\alpha_{n}\right\}$. The latter follows from Theorem 3, a somewhat stronger assertion.

Throughout, we shall point out various open questions.

Henceforth let $A$ be the collection of all positive, strictly 
decreasing functions defined on the positive integers whose elements $\alpha=\alpha_{n}=\alpha(n)$ satisfy (1.4). We call the function $C_{\alpha}(z)$ of (1.3) the Vieta product of $\alpha$. It is easily seen that $C_{\alpha}$ converges for all $\alpha \in A$, and that

$$
C_{\alpha}\left(z^{-1}\right)=-C_{\alpha}(z) .
$$

Let the fractional powers in (1.3) have their principal values. Then $C_{\alpha}(z)$ is holomorphic in the slit plane (that is, the complex plane with the closure of the negative real axis deleted).

In some sense (1.1) is four centuries old, since Vieta's formula

$$
\prod_{n=1}^{\infty} \cos \left(\theta / 2^{n}\right)=(\sin \theta) / \theta
$$

can be rewritten as

$$
i \theta=\left(e^{i \theta}-1\right) \prod_{n=1}^{\infty} \frac{2}{1+\exp \left(i \theta 2^{-n}\right)},
$$

and $\theta$ can be replaced by $-i \ln x$. M. Kac [9] relates (1.6) to expansions of real numbers in base 2 and uses it as the starting point of his well-known introduction to statistical independence. Infinite cosine products of the form

$$
\prod_{n=1}^{\infty} \cos \alpha_{n} \theta
$$

have been intensively studied in connection with Pisot-Vijayaraghavan numbers [12]. In fact, the product on the left of (1.6) plays an exceptional role in Theorem 2, p. 40 of [22]. For more about these products and their relation to certain digital problems, see [13].

From the point of view of [12] it is of interest to consider $C_{\alpha}(x)$ as defined on the "spiral staircase" Riemann surface that is the natural domain of $\ln z$. Since

$$
\prod_{n=1}^{\infty} \cos \left(\alpha_{n} \theta / 2\right)=i[2 \sin (\theta / 2)] C_{\alpha}^{-1}\left(e^{i \theta}\right),
$$

some of the problems discussed in [12] concerning Fourier-Stieltjes transforms of singular functions are related to the asymptotic behavior of $C_{\alpha}(z)$ as $z$ continuously winds about the branch point $z_{0}=0$.

We prove in $\S 2$ an identity generalizing (1.1). In $\S 3$ we show that under certain restrictions (including $\left.\alpha_{1} \leqq 1 / 2\right)$ the $C_{\alpha}(z)$ yield one-to-one conformal mappings of the slit plane onto a narrow strip. Thus they provide us with mappings qualitatively very similar to the mapping determined by $\ln z$; see Theorem 1 . The proof uses certain monotonicity properties of the Vieta products. In $\S 4$ we 
examine (see especially Corollary 4.1 of Theorem 2) how $C_{\alpha}(x)$ grows for $x$ real, $x \rightarrow+\infty$.

It seems natural to ask whether there is an $\alpha \neq \tau$ for which $C_{\alpha}$ is the product of $\ln z$ with a rational function. This is ruled out by Theorem 3 of $\S 5$, a uniqueness theorem for Vieta products. Such uniqueness theorems are very well known for power and Dirichlet series, and are established for a different kind of infinite product by J. F. Ritt [11]; see also [6]. The proof we present uses the Eulerian polynomials. Especially curious is the analytic function $G_{t}(\alpha)$ that the proof attaches to $C_{\alpha}(z)$; it is a sort of exponential generating function for certain "jumps" associated with $C_{\alpha}(z)$. We leave open the problem of when Vieta products exist for "approximately logarithmic" functions.

2. Elementary considerations. We first establish an identity with a parameter $s$ from which (1.1) and (1.6) follow by setting $s=2$. Then we examine the Taylor expansions of Vieta products about 1 .

Let

$$
\begin{aligned}
V(z, s)= & \left(z^{s}-1\right) \frac{s}{1+z+\cdots+z^{s-1}} \cdot \frac{s}{1+z^{1 / s}+\cdots+z^{(s-1) / s}} \\
& \times \frac{s}{1+z^{1 / s^{2}}+\cdots} \cdots \\
= & \left(z^{s}-1\right) \prod_{n=0}^{\infty} \frac{s\left(z^{s^{-n}}-1\right)}{z^{s^{-n+1}}-1}
\end{aligned}
$$

where $s \geqq 2$ is an integer. For $s=2$ this reduces to twice the right side of (1.1). It is easy to establish convergence, so $V(x)>0$ for $x>1$. Clearly $V(x)$ grows more slowly than any power of $x$, and

$$
V\left(x^{-1}\right)=-V(x), \quad V\left(x^{s}\right)=s V(x) .
$$

Proposition 2.1.

$$
V(z)=s \ln z .
$$

We first establish the following.

LEMMA. Let $U$ be a connected neighborhood of the strictly positive real axis. Let $0<r<1$ and let $f(z)$ be holomorphic on $U$. If $f(1)=0$ and

$$
f\left(x^{r}\right)=r \cdot f(x)
$$

for all $x>0$, then 


$$
f(z)=f^{\prime}(1) \ln z, \quad z \in U .
$$

Proof. For $z$ fixed and $n$ large, (2.4) and Taylor's theorem with remainder yield

$$
\begin{aligned}
r^{n} f(z) & =f\left[\exp \left(r^{n} \ln z\right)\right] \\
& =f\left[1+r^{n} \ln z+O\left(r^{2 n}\right)\right] \\
& =f^{\prime}(1)\left[r^{n} \ln z+O\left(r^{2 n}\right)\right]+(1 / 2) f^{\prime \prime}(\xi) O\left(r^{2 n}\right)
\end{aligned}
$$

where $.5<\xi<2$. Formula (2.5) follows for $z$ real upon dividing both sides of (2.6) by $r^{n}$ and letting $n \rightarrow \infty$. The result follows by analytic continuation.

To prove the proposition, take $r=s^{-1}$ to see that (2.5) is valid for $f=V$. To determine $V^{\prime}(1)$, note that by (2.1) and (2.5) we have

$$
\begin{aligned}
\ln V-\ln \ln x= & \ln \left[\left(x^{s}-1\right) / \ln x\right] \\
& +\sum_{n=1}^{\infty} \ln \left[s /\left(1+x^{s^{-n+1}}+\cdots+x^{(s-1) s^{-n+1}}\right)\right] .
\end{aligned}
$$

As $x \rightarrow 1$ the right hand side approaches $\ln s$, since every term of the infinite sum approaches 0 . This proves (2.3).

Upon replacing $z$ by $e^{i \theta}$, formula (2.3) reduces to the trigonometric identity

$$
\frac{\sin (s \theta / 2)}{s \theta / 2}=\prod_{n=1}^{\infty}\left\{\left(\sin \frac{s \theta}{2 s^{n}}\right) /\left(s \sin \frac{\theta}{2 s^{n}}\right)\right\} .
$$

This in turn reduces to Vieta's (1.6) when $s=2$.

Proposition 2.1 is in fact valid for any product

$$
V^{*}(z)=Q(z) \frac{P(1)}{P(z)} \frac{P(1)}{P\left(z^{1 / s}\right)} \frac{P(1)}{P\left(z^{2 / s}\right)} \cdots
$$

provided $P, Q=Q_{s}$ are "nice" functions satisfying $Q(1)=0, Q^{\prime}(1)=s$, and

$$
Q\left(z^{s}\right)=s Q(z) P\left(z^{s}\right) / P(1) .
$$

However, I know only the case $Q(z)=z^{s}-1$.

We now examine $C_{\alpha}(z)$, where

$$
\alpha_{n}=(k-1) k^{-n}
$$

and $k \geqq 2$ is an integer.

Proposition 2.2. Let $x>1$. If there is a constant $q$ such that

$$
C_{\alpha}(x)=q \ln x+o(\ln x) \text {, }
$$


then $k=2$.

Proof. Upon applying (2.12) to the identity

$$
C_{\alpha}\left(x^{k}\right)=2\left(x^{k}-1\right)\left(x^{k-1}+1\right)^{-1}(x-1)^{-1} C_{\alpha}(x)
$$

we obtain

$$
k+o(1)=2
$$

as $x \rightarrow \infty$, so $k=2$.

In general, however, $C=C_{\alpha}(z)$ does strongly resemble a logarithm in small neighborhoods of 1 . Clearly $C(1)=0$, and $C^{\prime}(1)=1$ follows from (1.3) upon dividing both sides by $z-1$ and letting $z \rightarrow 1$.

Set

$$
S=S(z)=\sum_{n=1}^{\infty} \frac{\alpha_{n} z^{\alpha_{n}-1}}{1+z^{\alpha_{n}}} .
$$

Logarithmic differentiation of (1.3) followed by subtraction of $C^{\prime}(1)$ yields

$$
\left[C^{\prime}(z)-C^{\prime}(1)\right]=\left[\frac{C(z)-C(1)}{z-1}-C^{\prime}(1)\right]-C(z) S(z) .
$$

Upon expanding both bracketed terms into Taylor series we obtain

$$
C S=\sum_{m=2}^{\infty} C^{(m)}(1)(z-1)^{m-1}\left[\frac{1}{m !}-\frac{1}{(m-1) !}\right] .
$$

By (2.17) and the Leibniz formula,

$$
\begin{aligned}
& \frac{-k}{k+1} C^{(k+1)}(1)=(C S)^{(k)}(1) \\
& =\sum_{j=1}^{k}\left(\begin{array}{c}
k \\
j
\end{array}\right) C^{(j)}(1) S^{(k-j)}(1), \quad k \geqq 1 .
\end{aligned}
$$

Since $S^{(0)}(1)=1 / 2$, equation (2.18) with $k=1$ yields

$$
C^{(2)}(1)=-1
$$

so every $C_{\alpha}(z)$ agrees with $\ln z$ up to second derivatives at $z=1$. The higher order derivatives have a more complicated appearance; for example

$$
\begin{aligned}
& C^{(3)}(1)=(3 / 4)\left(3-\sum \alpha_{n}^{2}\right) \\
& C^{(4)}(1)=(-3 / 2)\left(5-3 \sum \alpha_{n}^{2}\right) \\
& C^{(5)}(1)=(5 / 16)\left(105-86 \sum \alpha_{n}^{2}+3\left(\sum \alpha_{n}^{2}\right)^{2}+2 \sum \alpha_{n}^{4}\right)
\end{aligned}
$$


3. Vieta products as conformal maps. We begin by describing some very well-known properties of the logarithm, and then examine the extent to which they can be extended to arbitrary Vieta products. The function $w=\ln z$, where $z=x+i y$ and $w=$ $u+i v$, gives a conformal map from the $z$ plane to the $w$ plane. The region $R$ in the $z$ plane exterior to the unit circle $U$ and bounded by $U$ and the upper and lower lips $L_{1}, L_{2}$ of the ray $x \leqq-1$ maps in a one-to-one manner onto the strip $\sigma$ of width $2 \pi$ in the right half of the $w$ plane, bounded by the segment $I$ of the $v$ axis given by $-\pi \leqq v \leqq \pi$, and the lines $M_{1}$ and $M_{2}$ given by $v=\pi$ and $v=-\pi$ respectively. The boundaries $I, M_{1}, M_{2}$ correspond to $U$, $L_{1}, L_{2}$ respectively. This mapping "squeezes out angular variation"; in $R$ the argument varies from $-\pi$ to $\pi$, while (for $|z|$ large) its image is contained in

$$
-\varepsilon \leqq \arg w \leqq \varepsilon
$$

for any $\varepsilon>0$. At the same time $R$ is "contracted inwards uniformly"; a point $r$ units from the origin is taken to a point about $\log r$ units from the origin, regardless of its argument. Since

$$
\ln z^{-1}=-\ln z,
$$

the interior of the unit circle is mapped similarly onto the reflection of $\sigma$ in the $v$-axis.

Easiest to extend is the "uniform inwards contraction property", that asserts in particular that the gross behavior of the modulus of $C_{\alpha}(z)$ is determined by the modulus of $z$.

Proposition. Let $r>0$ be real, and $-\pi<\theta \leqq \pi$. Set

$$
q=q_{\alpha}(x, \theta)=C_{\alpha}(r) / C_{\alpha}\left(r e^{i \phi}\right) .
$$

Then

$$
K \frac{|r-1|\left|r^{\alpha_{1}}+\exp \left(i \theta \alpha_{1}\right)\right|}{\left|r-e^{i \theta}\right|\left|r^{\alpha_{1}}+1\right|} \leqq|q| \leqq \frac{|r-1|}{\left|r-e^{i \theta}\right|}
$$

where $K$ is an absolute constant. that

Proof. The upper bound is trivial. For the lower bound, note

$$
q=\frac{r-1}{e^{i \theta} r-1} \prod_{m=1}^{\infty} \frac{1+r^{\alpha_{m}} \exp \left(i \theta \alpha_{m}\right)}{1+r^{\alpha_{m}}}
$$

and that the square of the absolute value of each term in the product is 


$$
\begin{aligned}
1-2 r^{\alpha_{m}}\left(1+r^{\alpha_{m}}\right)^{-2}\left(1-\cos \alpha_{m} \theta\right) \\
\quad \geqq 1-\left(1-\cos \alpha_{m} \theta\right) / 2 \\
=\cos ^{2}\left(\alpha_{m} \theta / 2\right) .
\end{aligned}
$$

Since

$$
\cos \theta \geqq 1-\theta^{2} / 2
$$

we have the left inequality of (3.4) with

$$
\prod_{m=2}^{\infty}\left(1-\alpha_{m}^{2} \theta^{2} / 8\right)
$$

in place of $K$. Now $\sum \alpha_{m}^{2}$ is at most 1 and $\alpha_{m}$ is at most $1 / 2$ for $m \geqq 2$, so the infinite product (3.8) is bounded below by an absolute constant. This completes the proof.

Now set $z=r e^{i \theta}$ where $r>0$ and $-\pi<\theta \leqq \pi$, and let

$$
\delta(r, \theta)=1-2 r \cos \theta+r^{2}, \quad \delta(n, r, \theta)=1+2 r^{\alpha_{n}} \cos \theta \alpha_{n}+r^{2 \alpha_{n}} .
$$

By putting each term of the product $C_{\alpha}(z)$ into polar form, we find that

$$
C_{\alpha}(z)=\rho_{\alpha}(z) \exp \left[i \dot{\phi}_{\alpha}(z)\right]
$$

where

$$
\rho_{\alpha}(z)=\delta(r, \theta)^{1 / 2} \prod_{n=1}^{\infty} 2 \delta(n, r, \theta)^{-1 / 2}
$$

and

$$
\phi_{\alpha}(z)=\tan ^{-1} \frac{r \sin \theta}{r \cos \theta-1}-\sum_{n=1}^{\infty} \tan ^{-1} \frac{r^{\alpha_{n}} \sin \theta \alpha_{n}}{1+r^{\alpha_{n}} \cos \theta \alpha_{n}} ;
$$

here the inverse tangent is defined for $0 \leqq \theta \leqq \pi$ by

$$
\tan ^{-1} u= \begin{cases}\arctan u & u \geqq 0 \\ \pi+\arctan u & u<0\end{cases}
$$

and for $-\pi<\theta<0$ by

$$
\tan ^{-1} u= \begin{cases}\arctan u & u \leqq 0 \\ -\pi+\arctan u & u>0\end{cases}
$$

where $\arctan u$ denotes the principal value of the inverse tangent. From (3.11) and (3.12) we obtain

$$
r \frac{\partial \ln \rho}{\partial r}=\frac{\partial \phi}{\partial \theta}=\frac{r(r-\cos \theta)}{\delta(r, \theta)}-\sum_{n=1}^{\infty} \frac{\alpha_{n} r^{\alpha}{ }^{\alpha}\left(r^{\alpha_{n}}+\cos \theta \alpha_{n}\right)}{\delta(n, r, \theta)}
$$




$$
\frac{\partial \ln \rho}{\partial \theta}=-r \cdot \frac{\partial \phi}{\partial r}=\frac{r \sin \theta}{\delta(r, \theta)}+\sum_{n=1}^{\infty} \frac{\alpha_{n} r^{\alpha} \sin \theta \alpha_{n}}{\delta(n, r, \theta)} .
$$

We now study $\arg C_{\alpha}(z)$. From (3.13) and $\sum \alpha_{n}=1$ we obtain

$$
\frac{\partial \phi_{\alpha}(z)}{\partial \theta}=\frac{r \cos \theta-1}{\delta(r, \theta)}+\sum_{n=1}^{\infty} \frac{\alpha_{n}\left(r^{\alpha_{n}} \cos \theta \alpha_{n}+1\right)}{\delta(n, r, \theta)} .
$$

If $r=1$ this derivative is zero; in fact, by (1.9) the unit circle maps onto a segment of the imaginary axis containing the origin. Now the infinite sum on the right of (3.15) equals

$$
\sum_{n=1}^{\infty} \frac{\alpha_{n}}{r^{\alpha_{n}}+1}+\sum_{n=1}^{\infty} \frac{\alpha_{n} r^{\alpha_{n}}\left(1-r^{\alpha_{n}}\right)\left(1-\cos \theta \alpha_{n}\right)}{\left(1+r^{\alpha_{n}}\right) \delta(n, r, \theta)}
$$

and since $\cos \theta \alpha_{n} \geqq 0$ for $n \geqq 2$, we have

$$
\frac{\partial \phi_{\alpha}(z)}{\partial \theta}=O\left(r^{-1}\right)+O\left(r^{-\alpha_{1}}\right)+O\left(\sum_{n=2}^{\infty} \alpha_{n} r^{-\alpha_{n}}\right) .
$$

Since $\sum \alpha_{n}$ converges and $r^{-\alpha_{n}} \rightarrow 0$ as $r \rightarrow \infty$, it is clear that the above derivative is $o(1)$. Hence the Vieta product mapping will "squeeze out angular variation" in general. It is, however, not immediately clear when the analogues of $M_{1}$ and $M_{2}$ (the images of the upper and lower lips of $x \leqq-1$ ) will have bounded imaginary parts. We establish this in the case

$$
\alpha_{n}=c_{1} e^{-c_{2} n}
$$

where $c_{1}$ is positive and $c_{2}$ is at least $\ln 2$.

LEMMA. If $\alpha \in A$ where $\alpha_{n}$ is of the form (3.18), then there are constants $h_{1}, h_{2}$ such that

$$
h_{1}(\ln r)^{-1} \leqq \sum_{n=1}^{\infty} \alpha_{n} r^{-\alpha_{n}} \leqq h_{2}(\ln r)^{-1} .
$$

Proof. It is easily established by elementary calculus that the numbers $b_{n}=\alpha_{n} r^{-\alpha_{n}}$ increase to a maximum value as $n$ approaches

$$
c_{2}^{-1} \ln \ln r+O(1)
$$

and then decrease. Thus we find that the maximum value exceeds a constant multiple of $(\ln r)^{-1}$. We also see from this behavior that the sum is at most

$$
\begin{gathered}
\int_{0}^{\infty} c_{1} e^{-c_{2} t} \exp \left[-(\ln r) c_{1} e^{-c_{2} t}\right] d t+O(\ln r)^{-1} \\
\leqq c_{1} \int_{0}^{\infty} \exp \left[-(\ln r) c_{1} u\right] d u+O(\ln r)^{-1},
\end{gathered}
$$


and the result follows.

From (3.17), (4.9), and $-\pi<\theta \leqq \pi$ we have

$$
\phi_{\alpha}(z)=O(\ln r)^{-1}, \quad \rho_{\alpha}(z)=O\left[(\ln r)^{(\ln 2) / c_{2}}\right]
$$

so finally

$$
\operatorname{Im} C_{\alpha}(z)=O\left[(\ln r)^{\left[-c_{2}+\ln 2\right] / c_{2}}\right] .
$$

We shall use (3.13) and (3.14) to obtain more knowledge of the behavior of $C_{\alpha}(z)$ as $r$ and $\theta$ are varied individually. Results italicized below are of key importance in establishing Theorem 1 .

However, we shall first discuss logarithmic concavity on the real axis. In the course of doing this we shall discover (see (3.31)) that $C_{\alpha}(r)$ is strictly increasing for $r>1$, and hence for $r>0$ by (1.5). Note that the function

$$
q=q\left(r^{\circ}\right)=\ln \ln \mathrm{r},
$$

defined for $r>1$, satisfies

$$
q^{\prime \prime}+r^{-1} q^{\prime}=-(r \ln r)^{-2}<0 .
$$

In our case, (3.13) and (3.14) yield

$$
\frac{\partial^{2} \ln \rho}{\partial r^{2}}+r^{-2} \frac{\partial^{2} \ln \rho}{\partial \theta^{2}}+r^{-1} \frac{\partial \ln \rho}{\partial r}=0
$$

and

$$
\frac{\partial^{2} \dot{\phi}}{\partial \theta^{2}}+r^{2} \frac{\partial^{2} \dot{\varphi}}{\partial r^{2}}+r \frac{\partial \dot{\phi}}{\partial r}=0
$$

Since

$$
\begin{aligned}
\frac{\partial^{2} \ln \rho}{\partial \theta^{2}}= & \frac{r\left[2 r^{2} \cos 2 \theta-\left(1+r^{2}\right) \cos \theta\right]}{\hat{\delta}(r, \theta)^{2}} \\
& +\sum_{n=1}^{\infty} \frac{\alpha_{n}^{2} r^{\alpha_{n}}\left[2 r^{\alpha_{n}}+\left(1+r^{2 \alpha_{n}}\right) \cos \theta \alpha_{n}\right]}{\delta(n, r, \theta)^{2}}
\end{aligned}
$$

we see that at $\theta=0$ the above derivative, for $r>1$, is

$$
\frac{r(r+1)}{(r-1)^{3}}+\sum_{n=1}^{\infty} \frac{\alpha_{n}^{2} r^{\alpha_{n}}}{\left(1+r^{\alpha_{n}}\right)^{2}}>0
$$

Set

$$
Q(r)=\ln \rho(r)=\ln C_{\alpha}(r), \quad r>1 .
$$

We have, by (3.26) and (3.29), the inequality 


$$
\left(r Q^{\prime}\right)^{\prime}=Q^{\prime \prime}+r^{-1} Q^{\prime}<0 .
$$

Thus $r Q^{\prime}$ is decreasing, so if $Q^{\prime}(r)$ is ever nonpositive, it must remain nonpositive. This would imply that $Q(r)$ is bounded above. But this contradicts (4.25). Hence $Q\left(r^{\circ}\right)$ is strictly increasing for $r>1$, and from (3.31) we see that $Q^{\prime \prime}(r)$ is negative for $r>1$. Thus $\rho(r)$ is logarithmically concave. An open problem is to determine for what $\alpha$ we have actual concavity.

We now show that for

$$
\alpha_{1} \leqq 1 / 2
$$

the function $C_{\alpha}(z)$ has other monotonicity properties in common with In $z . \quad B y$ (3.14) the quantity $\ln \rho$ is strictly increasing as $\theta$ increases from 0 to $\pi$ and strictly decreasing as $\theta$ decreases from 0 to $-\pi$. Now consider $\ln \rho$ for $r>1$ and $0 \leqq \theta \leqq \pi$. Since

$$
\frac{d}{d a}\left(\frac{r+a}{r^{2}+2 a r+1}\right)=\frac{1-r^{2}}{\left(r^{2}+2 a r+1\right)^{2}}<0
$$

we have from (3.13) and $0 \leqq \theta \alpha_{n} \leqq \pi / 2$ that

$$
\begin{aligned}
r \frac{\partial \ln \rho}{\partial r^{r}} & \geqq \frac{r}{1+r}-\sum \frac{\alpha_{n} r^{2 \alpha_{n}}}{1+r^{2 \alpha_{n}}} \\
& =\sum_{n=1}^{\infty} \frac{\alpha_{n}}{1+r^{2 \alpha_{n}}}-\frac{1}{1+r}>0
\end{aligned}
$$

since the last sum in (3.34) is a weighted average of terms at least as large as $(1+r)^{-1}$, at least one of which strictly exceeds that quantity. Thus $\ln \rho$ is strictly increasing along every ray in the upper half plane that emanates from the origin. Since $C_{\alpha}(z)$ is real for $z=x>0$, we easily see that it has a strictly positive imaginary part when the same is true for $z \in R$ and that $C_{\alpha}(z)$ assumes conjugate values at conjugate points. Thus $\ln \rho$ is strictly increasing along every ray through the origin. It now follows from (3.13) and (3.14) that the argument of $C_{\alpha}(z)$ is strictly increasing in $\theta$, and strictly decreasing in $r$.

To show that $C_{\alpha}(z)$ is one-to-one when (3.32) is valid, it clearly suffices to show this for $z$ in the upper half plane $H$. Let $D$ denote a bounded simply connected open set in the complex plane. We require two lemmas, the first of which follows from the principle of the argument.

LEMMA. Let $f(z)$ be a nonconstant holomorphic function on $D$, and $J$ a positively oriented Jordan curve contained in $D$. If $f(z)$ is not one-to-one on the interior of $J$, then there is a point $z_{0}$ in $D$ such that the winding number of $f(J)$ with respect to $f\left(z_{0}\right)$ exceeds one. 
LEMma. Let $j(t)$, where $0 \leqq t \leqq 1$ and $j(0)=j(1)$, describe a positively oriented piecewise smooth closed curve in the open right half plane $K$. Say there is a point $p_{0}$ in $K$ such that the winding number of $J$ with respect to $p_{0}$ exceeds 1 . Then there does not exist a $t_{0}$ with $0<t_{0}<1$ such that arg $j(t)$ is nondecreasing for $0 \leqq t \leqq t_{0}$ and $|j(t)|$ is nonincreasing for $t_{0} \leqq t \leqq 1$.

Proof. Choose $0 \leqq t_{1} \leqq 1$ so that as $J$ is traversed, (i) $\arg \left[j(t)-p_{0}\right]$ has a net increase of $2 \pi m$ (where $m \geqq 1$ is integral) as $t$ increases from 0 to $t_{1}$, and (ii) the same holds for $t$ increasing from $t_{1}$ to 1 . Trivially $t_{0}$ is not interior to both intervals $\left[0, t_{1}\right]$ and $\left[t_{1}, 1\right]$. Now either $\arg j(t)$ or $|j(t)|$ will exhibit monotone behavior in the interval to which $t_{0}$ is not interior. But this is an obvious impossibility.

THEOREM 1. If (3.32) holds, the function $C_{\alpha}(z)$ is one-to-one on the slit complex plane.

Proof. It suffices to show this for $|z|>1$. Fix a small $\varepsilon>0$. Let $J^{*}$ be the positively oriented Jordan curve that starts at $-\varepsilon^{-1}+$ $i \varepsilon$ and moves parallel to the $x$ axis until it touches the unit circle, then goes clockwise around the part of the unit circle lying in $H$ until it reaches 1 , then moves along the $x$ axis to $\left(\varepsilon^{-2}+\varepsilon^{2}\right)^{1 / 2}$, whereupon it proceeds counterclockwise in a circle about 0 of this radius until it returns to its starting point. For $\varepsilon$ sufficiently small this curve will enclose any preassigned point $z$ in $H$ such that $|z|>1$. Clearly the image of $J^{*}$ by $C_{\alpha}(z)$ is a curve that passes through the origin. By the monotonicity properties italicized above we see that from the origin it moves along the $u$ axis to some point $u_{0}$, and then along a curve joining $u_{0}$ to some point $u_{1}+i v_{i}$ in such a way that its argument is nondecreasing. From $u_{1}+i v_{1}$ it moves to a point $H$ on the imaginary axis in such a way that its modulus is always decreasing. Once on the imaginary axis, it goes straight down to the origin. If $C_{\alpha}(z)$ were not one-to-one, this image, by the first lemma, would wind at least twice about some point. But this is ruled out by the second lemma, and the proof is completed.

Let $E_{\alpha}(z)$ denote the inverse of $C_{\alpha}(z)$; thus

$$
E_{\tau}(z)=e^{z} \text {. }
$$

An open question here is to determine those $\alpha \in A$ for which $E_{\alpha}(z)$ can be extended to an entire function.

4. Growth of Vieta products. The main result here is Theorem 2, an estimate on the size of a Vieta product. Note there is no claim that the "main terms" exceed the error terms. A slight 
modification of the argument will produce lower bounds; the Proposition following Theorem 2 asserts that every Vieta product tends to infinity along the real axis.

Let $A^{*}$ be the set of all $\alpha \in A$ that are restrictions of a twice continuously differentiable function $\alpha(t)$ defined for all $t>0$ such that

$$
\alpha^{\prime}(t)<0
$$

and

$$
\alpha^{\prime \prime}(t)>0
$$

for $t>0$. It is convenient to introduce the function

$$
S(x, \alpha, M)=\ln \left[\frac{x 2^{-M}}{x-1} C_{\alpha}(x)\right] .
$$

We can now state a general result on the growth of Vieta products.

THEOREM 2. Let $x \geqq 1$ and $\alpha \in A^{*}$. If $M$ is a positive integer such that

$$
0<\delta<\alpha(M) \ln x<1
$$

then

$$
S(x, \alpha, M)=1 / 2 \ln x \sum_{m>M} \alpha(m)+O[E(x, M, \delta)]
$$

where

$$
\begin{aligned}
E(x, M, \delta)= & \ln ^{2} x \sum_{m>M} \alpha^{2}(m)+O(1) \\
& +O\left[\left(\left|\alpha^{\prime}(M)\right| \delta \ln x\right)^{-1}\right] .
\end{aligned}
$$

We shall apply this to the special cases

$$
\alpha(t)=c_{1} e^{-c_{2} t}
$$

and

$$
\alpha(t)=c_{1} t^{-b}
$$

where $c_{1}, c_{2}$ are positive constants, and $b$ is a constant exceeding 1 . Clearly each $\alpha$ belongs to $A^{*}$ for the appropriate normalizing constant $c_{1}$. Carlson's product (1.1) corresponds to (4.7) in the special case $c_{1}=1$ and $c_{2}=\ln 2$.

CoRollary 4.1. Let $x$ be a large real number. For the $\alpha$ of (4.7) there are positive constants $d_{1}, d_{2}$ such that 


$$
d_{1}(\ln x)^{(\ln 2) / c_{2}} \leqq(x-1) \prod_{m=1}^{\infty} \frac{2}{1+x^{\alpha_{m}}} \leqq d_{2}(\ln x)^{(\ln 2) / c_{2}}
$$

Proof. Choose $M$ to be the greatest integer in $c_{2}^{-1} \ln \ln x$. Then every term on the right of (4.5) and (4.6) is bounded, and the result follows.

CoRollary 4.2. Let $x$ be a large real number. For the $\alpha$ of (4.8) there is a positive constant $c_{3}=c_{3}(b)$ such that

$$
C_{\alpha}(x) \leqq \exp \left[c_{3}(\ln x)^{1 / b}\right] \text {. }
$$

Proof. Choose $M$ to be the greatest integer in $(\ln x)^{1 / 6}$. Then $x^{-\alpha(M)}$ is bounded, and every other individual term on the right of (4.5) and (4.6) has order of magnitude

$$
(\ln x)^{1 / b},
$$

so the upper bound of (4.10) follows.

In each case, the idea was to let $M$ be the greatest integer in

$$
\alpha^{-1}(1 / \ln x)
$$

where $\alpha^{-1}$ is the function inverse to $\alpha$.

To prove Theorem 2, write

$$
S(x, \alpha, M)=-S_{1}(x, \alpha, M)+S_{2}(x, \alpha, M)
$$

where

$$
S_{1}=\sum_{m \supseteq M} \ln \left(1+x^{-\alpha(m)}\right)
$$

and

$$
\begin{aligned}
S_{2} & =-\sum_{m>M} \ln \left[1-1 / 2\left(1-x^{-\alpha(m)}\right)\right] \\
& =1 / 2 \sum_{m>M}\left(1-e^{-\alpha_{m} \ln x}\right)+O \sum_{m>M}\left[e^{-\alpha_{m} \ln x}-1\right]^{2} \\
& =1 / 2 \ln x \sum_{m>M} \alpha(m)+O \sum_{m>M} \alpha^{2}(m) \ln ^{2} x .
\end{aligned}
$$

Here we have made use of the fact that there are absolute constants $h_{1}, h_{2}>0$ such that

$$
|\ln (1+u)-u|<h_{1} u^{2}
$$

for

$$
-1 / 2 \leqq u \leqq 0
$$

and

$$
\max \left\{\left(e^{-t}-1+t\right),\left(e^{-t}-1\right)^{2}\right\}<h_{2} t^{2}
$$


for $0 \leqq t$. Next,

$$
S_{1}=S_{3}+S_{4}
$$

where

$$
0>S_{3}=\sum_{m \leqq M}\left\{\ln \left(1+x^{-\alpha_{m}}\right)-x^{-\alpha_{m}}\right\} \geqq\left(e^{-\bar{o}}-1\right)^{-1} \sum_{m \leqq M} x^{-2 \alpha_{m}}
$$

and

$$
S_{4}=\sum_{m \leq M} x^{-\alpha_{m}}=\int_{1}^{M} x^{-\alpha(t)} d t+O\left(x^{-\alpha(M)}\right) .
$$

Here the right side of (4.20) was obtained by majorizing the series for the logarithm by a geometric series. To estimate the integral on the right of (4.21) we observe that there is a continuously differentiable function $g(u)$ such that

$$
g(\alpha(t))=-\alpha^{\prime}(t) .
$$

By making the change of variable $u=\alpha(t)$, the integral becomes

$$
\int_{\alpha(M)}^{\alpha(1)} \exp [-(\ln x) u] \frac{d u}{g(u)} .
$$

By the intermediate value theorem the integral equals

$$
-\frac{\left[x^{-\alpha(M)}-x^{-\alpha(1)}\right]}{\alpha^{\prime}\left(M_{1}\right) \ln x} \leqq \frac{x^{-\alpha(M)}}{\left|\alpha^{\prime}(M)\right| \ln x}
$$

where $1 \leqq M_{1} \leqq M$; the upper bound of (4.24) follows from (4.1) and (4.2). The estimate (4.24) also applies to the sum on the right of (4.20) with $x^{2}$ in place of $x$. The theorem now follows from (4.15), (4.20), (4.21), and (4.24).

Proposition. For $\alpha \in A$ we have

$$
C_{\alpha}(x) \longrightarrow \infty \text { as } x \longrightarrow \infty \text {. }
$$

Proof. Set $M=0 . \quad$ By

$$
-\ln (1-u)>u
$$

and (4.15) we have

$$
S_{2} \geqq .5 \sum^{*}\left(1-x^{-\alpha(m)}\right) \geqq .5\left(1-e^{-u}\right) N(u, x)
$$

where the asterisk indicates the summation is limited to

$$
u /(\ln x) \leqq \alpha(m), \quad 2 \leqq m,
$$

and $N(u, x)$ is the number of $m$ for which (4.28) is valid. For any 
fixed $u>0$ it is clear from (1.4) that

$$
N(u, x) \longrightarrow \infty \text { as } x \longrightarrow \infty,
$$

and (4.25) follows.

5. Uniqueness. Let $L=L_{z}$ be the differential operator

$$
L=z \frac{d}{d z} .
$$

Let $T=T_{\alpha}$ be the operator that replaces $z$ by $z^{\alpha}$; for example $T_{3} \sin z=\sin z^{3}$. It is straightforward to show that

$$
L^{s} f\left(z^{\alpha}\right)=\alpha^{s} T L^{s} f(z), \quad s=1,2,3, \cdots
$$

for all infinitely differentiable functions $f(z)$.

Set $A_{1}(z)=z$, and for $n \geqq 1$ let

$$
\frac{A_{n+1}(z)}{(1-z)^{n+2}}=L\left[\frac{A_{n}(z)}{(1-z)^{n+1}}\right] \text {. }
$$

The polynomials $A_{1}(z)=z, \quad A_{2}(z)=z+z^{2}, \quad A_{3}(z)=z+4 z^{2}+z^{3}, \cdots$ are called the Eulerian polynomials. They may also be defined by

$$
A_{n}=A_{n}(z)=z(z-1)^{n} H_{n}(z)
$$

where

$$
\frac{1-z}{e^{t}-z}=\sum_{n=0}^{\infty} H_{n}(z) \frac{t^{n}}{n !} .
$$

For surveys of their properties see $[1,3,7,8]$. They satisfy the reciprocal property

$$
z^{n+1} A_{n}\left(z^{-1}\right)=A_{n}(z)
$$

and the recurrence

$$
A_{n+1}=(n+1) z A_{n}+z(1-z) \frac{d}{d z} A_{n} .
$$

From (5.7) we see that

$$
A_{n}(1)=n !
$$

and (by establishing recurrence relations for the individual coefficients of the $A_{n}$ ) that the coefficients of the polynomial $A_{n}$ are nonnegative; see [1]. Somewhat deeper is the fact that for $k \geqq 1$ we have

$$
A_{2 k}(-1)=0, \quad A_{2 k-1}(-1)=(-1)^{k-1} T_{k}
$$

where 


$$
T_{k}=(-1)^{k-1}(2 k)^{-1} B_{2 k}\left(2^{2 k}-1\right) 2^{2 k}
$$

and

$$
B_{2 k}=(-1)^{k-1} \frac{2(2 k) !}{(2 \pi)^{2 k}} \zeta(2 k)
$$

where $\zeta(s)$ is the Riemann zeta function; see [7], p. 2 and pp. 84-90, and [10], pp. 16-20. The elucidation of the asymptotic behavior of the Eulerian polynomials has been carried out by Evans [4]. He proves and applies the identity

$$
A_{n}(z)=n !(z-1)^{n+1} \sum_{m=-\infty}^{\infty}(\ln z+2 \pi i m)^{-n-1}
$$

valid for all complex $z \neq 0$ (the point $z=1$ is a removable singularity; note (5.8)).

We can now establish a uniqueness result for Vieta products. In what follows $U$ shall denote an open set containing the unit circle, and $z^{\alpha}$ shall denote the principal value of the fractional power.

THEOREM 3. Let $\alpha, \beta \in A$ and let $r(z)$ be a function meromorphic on $U$. If

$$
\prod_{m=1}^{\infty} \frac{2}{1+z^{\alpha_{m}}}=r(x) \prod_{m=1}^{\infty} \frac{2}{1+z^{\beta_{m}}}
$$

then $r(z)=1$ and

$$
\alpha_{m}=\beta_{m}, \quad m=1,2,3, \cdots .
$$

Proof. Logarithmic differentiation on $U$ yields

$$
\sum_{m=1}^{\infty} \frac{\alpha_{m} z^{\alpha_{m}}}{1+z^{\alpha_{m}}}=Q(z)+\sum_{m=1}^{\infty} \frac{\beta_{m} z^{\beta_{m}}}{1+z^{\beta_{m}}}
$$

where

$$
Q(z)=-z r^{\prime}(z) / r(z)
$$

Since $\sum \alpha_{m}$ and $\sum \beta_{m}$ converge, both sums in (5.15) are holomorphic in the plane with the closure $E$ of the negative real axis deleted. Moreover, since both sums are bounded in any disc, the function $r(x)$ can have neither zeros nor poles in $U$, and $Q(x)$ is holomorphic in the plane with $E-U$ deleted.

Apply the operator $L^{s}$ to both sides of (5.15). By uniform convergence, (5.2), and (5.3) we obtain 


$$
\begin{aligned}
& \sum_{m=1}^{\infty} \alpha^{s} A_{s}\left(-z^{\alpha}\right)\left(1+z^{\alpha}\right)^{-s-1} \\
& =-L^{s} Q(z)+\sum_{m=1}^{\infty} \beta^{s} A_{s}\left(-z^{\beta}\right)\left(1+z^{\beta}\right)^{-s-1}
\end{aligned}
$$

where $\alpha=\alpha_{m}, \beta=\beta_{m}$.

We now compute the jump in the values of the functions on the left and right of (5.17) as the negative real axis is crossed counterclockwise on the unit circle. With the aid of (5.6) we find that each coefficient of $\alpha^{8}$ on the left contributes

$$
\begin{aligned}
& A_{s}\left(-e^{i \pi \alpha}\right)\left(1+e^{i \pi \alpha}\right)^{-s-1}-A_{s}\left(-e^{-i \pi \alpha}\right)\left(1+e^{-i \pi \alpha}\right)^{-s-1} \\
& \quad=A_{s}\left(-e^{i \pi \alpha}\right)\left[\left(1+e^{i \pi \alpha}\right)^{-s-1}-\left(-e^{i \pi \alpha}\right)^{-s-1}\left(1+e^{-i \pi \alpha}\right)^{-s-1}\right] \\
& \quad=A_{s}\left(-e^{i \pi \alpha}\right)\left[1+(-1)^{s}\right]\left(1+e^{i \pi \alpha}\right)^{-s-1}
\end{aligned}
$$

Set

$$
u=u_{m}=-e^{i \pi \alpha}
$$

so $u \rightarrow-1$ as $m \rightarrow \infty$. Since $L^{s} Q(z)$ is continuous on $U$, we obtain, upon equating the jumps,

$$
F_{s}(\alpha)=F_{s}(\beta)
$$

where

$$
\begin{aligned}
F_{s}(\alpha) & =\sum_{m=1}^{\infty} \frac{1+(-1)^{s}}{1-u} \frac{\alpha^{s} A_{s}(u)}{(1-u)^{s}} \\
& =\sum_{m=1}^{\infty} \frac{u}{1-u}\left[1+(-1)^{s}\right] \alpha^{s} H_{s}(u) ;
\end{aligned}
$$

recall (5.4). Clearly the series converges for each $s$. We wish to multiply both sides of (5.21) by $t^{s} / s$ ! and sum over all $s \geqq 1$. Observe that

$$
\left|A_{s}(u)\right| \leqq s !
$$

by (5.8) and the remark following it. Hence for any bounded region in the $t$-plane and a suitably large integer $m_{0}$ the resulting double sum has the form

$$
\sum_{s} \sum_{m<m_{0}}+\sum_{s} \sum_{m \leq m_{0}}
$$

where the second sum is majorized by a constant multiple of the convergent series

$$
\sigma=\sum_{m \geqq m_{0}} \sum_{s \geqq 1} \alpha^{s}|t|^{s}=\sum_{m \geqq m_{0}} \frac{\alpha|t|}{1-\alpha|t|} .
$$


Here $\sigma$ converges throughout the region since $t$ is bounded and $\alpha=\alpha_{m}$ is very small for $m \geqq m_{0}$. This shows that the summations over $s$ and $m$ may be done in either order, and it follows from (5.5) and (5.20) that

$$
G_{t}(\alpha)=G_{t}(\beta)
$$

where

$$
G_{t}(\alpha)=\sum_{m=1}^{\infty} \frac{u}{1-u}\left[\frac{1-u}{e^{\alpha t}-u}+\frac{1-u}{e^{-\alpha t}-u}-2\right] .
$$

The series for $G_{t}(\alpha)$ is analytic as a function of $t$ except for simple poles at the zeros of $E_{1}(t)$ and $E_{2}(t)$ where

$$
E_{1}(t)=e^{\alpha t}+e^{i \pi \alpha}, \quad E_{2}(t)=e^{-\alpha t}+e^{i \pi \alpha} .
$$

The pole of $G_{t}(\alpha)$ closest to but above the real axis is

$$
i \pi\left(-1+\alpha(1)^{-1}\right) \text {; }
$$

from the corresponding statement for $G_{t}(\beta)$ we deduce that $\alpha(1)=$ $\beta(1)$. By subtracting off the $m=1$ terms from $G_{t}(\alpha), G_{t}(\beta)$ and repeating the argument we find that $\alpha(2)=\beta(2)$, and so forth. This proves the theorem.

We leave open the problem of the linear independence of three or more Vieta products over the field of functions meromorphic on $U$, and also the inhomogeneous problem for two products.

\section{REFERENCES}

1. L. Carlitz, Eulerian numbers and polynomials, Math. Mag., 32 (1959), 247-260.

2. B. C. Carlson, The logarithmic mean, Amer. Math. Monthly, 79 (1972), 615-618.

3. L. Comtet, Advanced Combinatorics, D. Reidel, Dordrecht, 1970.

4. R. Evans, An asymptotic formula for extended Eulerian numbers, Duke Math. J., 41 (1974), 161-175.

5. J. M. Feld, The expansion of analytic functions in a generalized Lambert series, An. nals of Math., 33 (1932), 139-143.

6. J. M. Feld and P. Newman, On the representation of analytic functions of several var. iables as infinite products, Bull. Amer. Math. Soc., (2) 36 (1930), 284-288.

7. D. Foata and M. -P. Schützenberger, Théorie Géométrique des Polynômes Eulériens, Springer-Verlag, Berlin, 1970.

8. G. Frobenius, Über die Bernoullischen und die Eulerschen Polynome, Sitzungsberichte der Preussische Akademie der Wissenschaften, (1910), 809-847.

9. M. Kac, Statistical Independence in Probability, Analysis, and Number Theory, John Wiley, New York, 1959.

10. H. Rademacher, Topics in Analytic Number Theory, Springer-Verlag, New York, 1973.

11. J. F. Ritt, Representation of analytic functions as infinite products, Math. Zeit., 32 (1930), 1-3.

12. R. Salem, Algebraic Numbers and Fourier Analysis, D. C. Heath, Boston, 1963. 
13. H. G. Senge and E. G. Straus, PV-numbers and sets of multiplicity, Periodica Math., 3 (1973), 93-100.

Received September 17, 1979.

UNIVERSITY OF ILLINOIS

UrBANA, IL 61801 



\section{PACIFIC JOURNAL OF MATHEMATICS}

\section{EDITORS}

DONALD BABBITT (Managing Editor)

University of Galifornia

Los Angeles, California 90024

Hugo RossI

University of Utah

Salt Lake City, UT 84112

C. C. MoOre AND ANDrew OGG

University of California

Berkeley, CA 94720

\section{J. DugundjI}

Department of Mathematics University of Southern California

Los Angeles, California 90007

R. Finn and J. Milgram

Stanford University

Stanford, California 94305

ASSOCIATE EDITORS

E. F. Beckenbachi

B. H. NeumanN

F. WOLF

K. YOSHIDA

\section{SUPPORTING INSTITUTIONS}

UNIVERSITY OF BRITISH COLUMBIA

CALIFORNIA INSTITUTE OF TECHNOLOGY

UNIVERSITY OF CALIFORNIA

MONTANA STATE UNIVERSITY

UNIVERSITY OF NEVADA, RENO

NEW MEXICO STATE UNIVERSITY

OREGON STATE UNIVERSITY

UNIVERSITY OF OREGON
UNIVERSITY OF SOUTHERN CALIFONIA

STANFORD UNIVERSITY

UNIVERSITY OF HAWAII

UNIVERSITY OF TOKYO

UNIVERSITY OF UTAH

WASHINGTON STATE UNIVERSITY

UNIVERSITY OF WASHINGTON

Printed in Japan by International Academic Printing Co., Ltd., Tokyo, Japan 


\section{Pacific Journal of Mathematics \\ Vol. 89 , No. 1 \\ May, 1980}

David Bressoud, A note on gap-frequency partitions ................ 1

John David Brillhart, A double inversion formula ................. 7

Frank Richard Deutsch, Günther Nürnberger and Ivan Singer, Weak

Chebyshev subspaces and alternation .......................

Edward Richard Fadell, The relationship between Ljusternik-Schnirelman

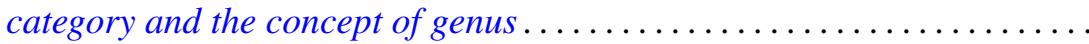

Harriet Jane Fell, On the zeros of convex combinations of polynomials.......

John Albert Fridy, An addendum to: "Tauberian theorems via block

dominated matrices" ..................................

Andrzej Granas, Ronald Bernard Guenther and John Walter Lee, Applications of topological transversality to differential equations. I. Some nonlinear

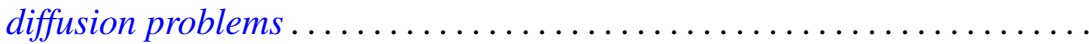

David E. Handelman and G. Renault, Actions of finite groups on self-injective

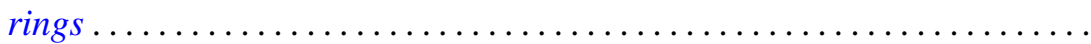

Michael Frank Hutchinson, Local $\Lambda$ sets for profinite groups .............

Arnold Samuel Kas, On the handlebody decomposition associated to a

Lefschetz fibration...

Hans Keller, On the lattice of all closed subspaces of a Hermitian space.....

P. S. Kenderov, Dense strong continuity of pointwise continuous

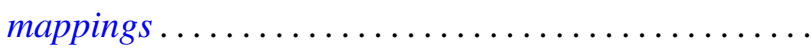

Robert Edward Kennedy, Krull rings.................

Jean Ann Larson, Richard Joseph Laver and George Frank McNulty,

Square-free and cube-free colorings of the ordinals ...

Viktor Losert and Harald Rindler, Cyclic vectors for $L^{p}(G)$

John Rowlay Martin and Edward D. Tymchatyn, Fixed point sets of

1-dimensional Peano continua...

Augusto Nobile, On equisingular families of isolated singularities ...

Kenneth Joseph Prevot, Imbedding smooth involutions in trivial bundles ...

Thomas Munro Price, Spanning surfaces for projective planes in four space.

Dave Riffelmacher, Sweedler's two-cocycles and Hochschild cohomology....

Niels Schwartz, Archimedean lattice-ordered fields that are algebraic over

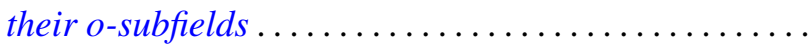

Chao-Liang Shen, A note on the automorphism groups of simple dimension groups.

Kenneth Barry Stolarsky, Mapping properties, growth, and uniqueness of

Vieta (infinite cosine) products ...

Warren James Wong, Maps on simple algebras preserving zero products. I.

The associative case ............................. 